ISSN 0103-9954

\title{
EXPORTAÇÃO E REPOSIÇÃO NUTRICIONAL NO PRIMEIRO DESBASTE DE UM POVOAMENTO DE Pinus taeda L. EM ÁREA DE SEGUNDA ROTAÇÃO
}

\author{
EXPORT AND NUTRITIONAL REPLACEMENT IN THE FIRST THINNING OF Pinus taeda L. IN A \\ SECOND ROTATION AREA
}

Eduardo Kneipp Londero ${ }^{1}$ Mauro Valdir Schumacher ${ }^{2}$ Denise Andréia Szymczak ${ }^{3}$ Márcio Viera ${ }^{4}$

\section{RESUMO}

O trabalho tem por objetivo quantificar a biomassa, exportação e reposição dos nutrientes em árvores do primeiro desbaste de um povoamento de Pinus taeda, em Cambará do Sul, RS. Para isso, foi realizado um inventário em três parcelas $(60 \mathrm{~m} \times 60 \mathrm{~m})$, sendo abatidas 3 árvores por parcela. Foram mensuradas acículas vivas e mortas, galhos vivos e mortos, casca, madeira comercial e madeira do ponteiro com casca. A biomassa totalizou $44,5 \mathrm{Mg} \mathrm{ha}^{-1}$, com $62,8 \%$ de madeira, $10,3 \%$ de galhos vivos, $8,9 \%$ de casca, $10,1 \%$ de acículas vivas, $6,1 \%$ de galho morto, $3,5 \%$ de ponteira com casca e $0,5 \%$ de acícula morta. A quantidade total de nutrientes na biomassa, em $\mathrm{kg} \mathrm{ha}^{-1}$, foi de: $100,5 \mathrm{de} \mathrm{N} ; 51,1$ de Ca; 46,8 de K; 18,2 de S; 14,3 de $\mathrm{Mg} ; 11,7$ de P; 0,599 de Mn; 0,158 de Fe; 0,435 de Zn; 0,181 de B e 0,177 de Cu. A quantidade de adubos a serem repostos dependerá do tipo da exploração. Se ocorrer remoção total da biomassa, a quantidade de adubos a ser reposto será de $391 \mathrm{~kg} \mathrm{ha}^{-1}$, e se considerar apenas a retirada da madeira esta reposição será aproximadamente $60 \%$ inferior, $169 \mathrm{~kg} \mathrm{ha}^{-1}$.

Palavras-chave: exportação de nutrientes; tipos de colheita; formas de manejo.

\begin{abstract}
This study aimed to quantify biomass, the export and nutrient replacement in trees of a first thinning Pinus taeda L. stand, in Cambará do Sul, RS state. To do so, a forest inventory was done in three plots $(60 \mathrm{~m} \mathrm{x}$ $60 \mathrm{~m}$ ), where three trees were cut in each plot. Live and dead needles, live and dead branches, bark, wood (bole) and wood from tip with bark, were measured. Total biomass was $44,5 \mathrm{Mg} \mathrm{ha}^{-1}$, being $62,8 \%$ of bole wood, $10,3 \%$ live branches, $8,9 \%$ bark, $10,1 \%$ live needles, $6,1 \%$ dead branches, $3,5 \%$ of wood from tip with bark and 0,5\% dead needles. Total nutrients in biomass, in $\mathrm{kg} \mathrm{ha}^{-1}$, was: 100,5 of N; 51,1 of Ca; 46,8 of $\mathrm{K} ; 18,2$ of S; 14,3 of Mg; 11,7 of P; 0,599 of Mn; 0,158 of Fe; 0,435 of Zn; 0,181 of B and 0,177 of Cu. The quantity of the fertilizer to be replaced will depend on the exploration type. If the total biomass occurs, the quantity of the fertilizers to be replaced will be of $391 \mathrm{~kg} \mathrm{ha}^{-1}$, and if it is considered just the wood harvest this replacement will be $60 \%$ inferior, $169 \mathrm{~kg} \mathrm{ha}^{-1}$.
\end{abstract}

Keywords: nutrients export; harvest types; management ways.

1. Engenheiro Florestal, Mestrando do Programa de Pós-graduação em Engenharia Florestal, Departamento de Ciências Florestais, Centro de Ciências Rurais, Universidade Federal de Santa Maria, Av. Roraima, 1000, CEP 97105-900, Santa Maria (RS). Bolsista CAPES. eklondero@gmail.com

2. Engenheiro Florestal, Dr. nat. techn., Professor do Departamento de Ciências Florestais, Centro de Ciências Rurais, Universidade Federal de Santa Maria, Av. Roraima, 1000, CEP 97105-900, Santa Maria (RS). Bolsista do CNPq.mvschumacher@gmail.com

3. Engenheira Florestal, Mestranda do Programa de Pós-graduação em Engenharia Florestal, Centro de Ciências Rurais, Universidade Federal de Santa Maria, Av. Roraima, 1000, CEP 97105-900, Santa Maria (RS). Bolsista CAPES.deniseszymczak@gmail.com

4. Engenheiro Florestal, Doutorando do Programa de Pós-graduação em Engenharia Florestal, Centro de Ciências Rurais, Universidade Federal de Santa Maria, Av. Roraima, 1000, CEP 97105-900, Santa Maria (RS). Bolsista CNPq. vieraflorestal@yahoo.com.br

Recebido para publicação em 3/03/2010 e aceito em 2/07/2010 


\section{INTRODUÇÃO}

As atividades silviculturais com espécies do gênero Pinus foram intensificadas a partir da segunda metade da década de sessenta, após a promulgação da lei dos incentivos fiscais. Extensas áreas foram ocupadas predominantemente com Pinus taeda e Pinus elliotti var. elliotti, constituindo hoje bases de importantes atividades industriais como produção de celulose e papel, embalagens, aglomerados, mobiliário, compensados, chapas dentre outras.

A implantação desses maciços florestais puros é devido à grande quantidade de matériaprima demandada para o uso em escala industrial. As práticas silviculturais aplicadas nesses maciços podem causar redução no estoque de nutrientes, comprometendo a produtividade contínua do ecossistema no decorrer das rotações de cultivo (BARICHELLO, 2003).

Com a intensificação das técnicas de manejo das plantações de pinus no sul do Brasil, está ocorrendo aumento na demanda de nutrientes pelas plantas, a qual poderá não ser satisfeita temporal e espacialmente pelo solo, requerendo uma suplementação de nutrientes por meio de fertilizantes (BARROS FILHO, 2003). Segundo Freitas (2000), em ecossistemas de florestas plantadas e naturais, a ciclagem de nutrientes configura-se como um fator essencial para avaliar o papel do sistema de manejo adotado, em favor do uso sustentado desses sistemas naturais, conjuntamente com a do sistema silvicultural mais apropriado para a manutenção da produtividade.

Devido a isso, o manejo eficiente de uma plantação florestal está ligado à ciclagem de nutrientes, para que ocorra um fluxo contínuo entre o que é depositado no solo com a queda do folhedo e o que é assimilado novamente pelas raízes. Portanto, estimar a exportação de macro e micronutrientes através dos diferentes componentes é importante para a compreensão de um manejo conveniente (LA TORRACA et al., 1984).

Em várias plantações florestais estabelecidas em solos de baixa fertilidade, constata-se a queda de produtividade ao longo de sucessivas rotações com a mesma espécie. Geralmente, a este fato está associado um declínio da fertilidade do solo, causado por um manejo inadequado, que inclui práticas de conservação e preparo de solo, fertilização insuficiente ou desbalanceada, e manejo impróprio dos resíduos culturais. O quadro torna-se mais grave quando são usados materiais genéticos melhorados, como os plantios monoclonais, com alta capacidade de extração de nutrientes (STAPE, 2002).

A exportação de nutrientes pela colheita florestal, segundo Ferreira et al. (2001) seria um dos fatores a ser considerado devido à preocupação com a manutenção da produtividade dos sítios. Principalmente em condições de baixo suprimento de elementos essenciais às árvores pelo solo, sendo que explorações intensivas em rotações curtas, sem previsão de um período mínimo necessário para reposição de nutrientes, têm sido apontadas como as maiores responsáveis pelo exaurimento do solo.

Aquantificação da biomassa e da exportação de nutrientes em qualquer forma de ecossistema florestal é de fundamental importância para que se conheça a dinâmica dos nutrientes nos diversos compartimentos do ecossistema, podendo-se assim, encontrar indicadores de possíveis impactos que algumas técnicas silviculturais podem causar. Como exemplo, pode-se citar os desbastes, que às vezes são realizados retirando toda a biomassa desbastada do interior do povoamento, seja a madeira para a indústria de celulose e papel, bem como os resíduos para a combustão em caldeiras, tendo assim, a exportação total de nutrientes do povoamento, prejudicando desta maneira, a ciclagem de nutrientes.

O trabalho teve por objetivo quantificar a biomassa, exportação e a reposição nutricional dos nutrientes contidos na biomassa do primeiro desbaste, de um povoamento de Pinus taeda L., em área de segunda rotação, no município de Cambará do Sul, RS.

\section{MATERIAIS E MÉTODOS}

\section{Caracterização da área experimental}

O estudo foi realizado em um povoamento de Pinus taeda, localizado no município de Cambará do Sul, situado nas coordenadas geográficas centrais de $28^{\circ} 53^{\prime} 07,2^{\prime \prime}$ de latitude Sul e $50^{\circ} 08^{\prime} 03,9$ ' de longitude Oeste, numa altitude média de $1100 \mathrm{~m}$ (Figura 1). A cidade de Cambará do Sul localizase no nordeste do Estado do Rio Grande do Sul, na região fisiográfica dos Campos de Cima da Serra.

$\mathrm{O}$ clima da região é o temperado, $\mathrm{Cfb}$ segundo a classificação de Köppen, com temperatura média anual de $15,5^{\circ} \mathrm{C}$, sendo $20,9^{\circ} \mathrm{C}$ para as máximas, $10,9^{\circ} \mathrm{C}$ para as mínimas, um extremo para as mínimas absolutas de $-7,2^{\circ} \mathrm{C}$, observados

Ci. Fl., v. 21, n. 3, jul.-set., 2011 


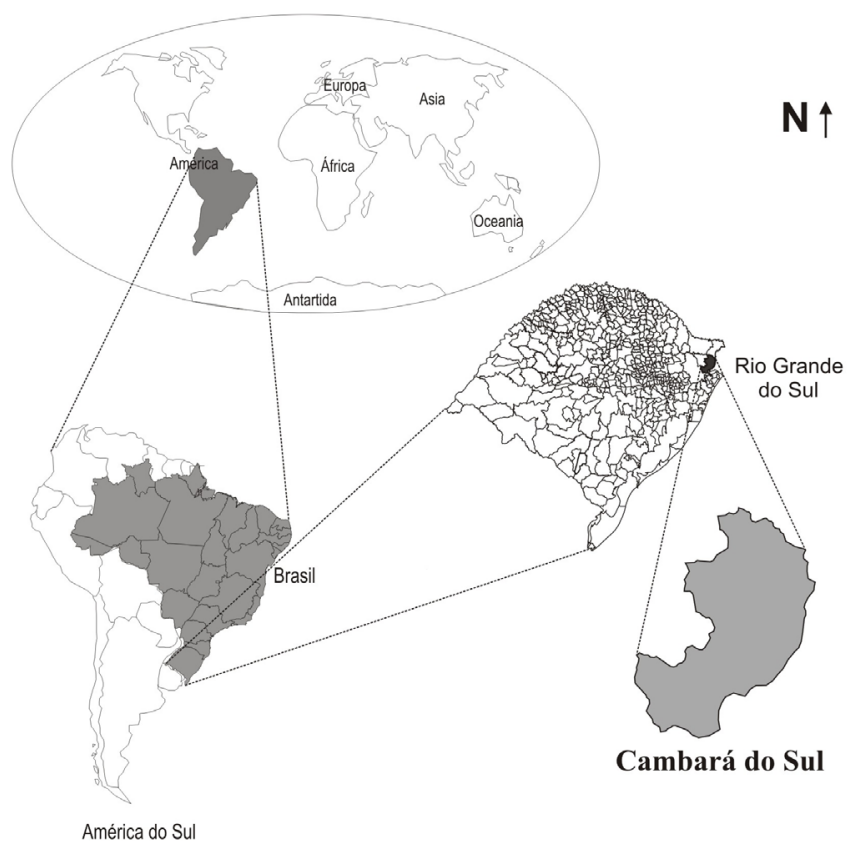

FIGURA 1: Localização do município de Cambará do Sul, RS.

FIGURE 1: Location of Cambará do Sul, RS state.

na estação meteorológica de Cambará do Sul, no período de 1997-2007 (INMET, 2008). Conforme Motta et al. (1971), os ventos dominantes na região são alísios, ou seja, sopram do mar para a terra, devido à região situar-se próximo ao litoral norte do estado do Rio Grande do Sul.

Quanto ao solo, o material de origem é rocha basáltica, resultante do derrame basáltico do Triássico Superior, predominando solos rasos com horizonte A de coloração escura, com baixa saturação de bases e teor elevado de alumínio trocável. O solo da região pertence à Unidade de Mapeamento Bom Jesus e é classificado como CAMBISSOLO HÚMICO Alumínico típico (STRECK et al., 2008).

O povoamento estudado foi implantado em área de corte raso, onde os resíduos do primeiro povoamento (Pinus taeda L.) foram enleirados. Para o plantio, utilizou-se coroamento $(60 \mathrm{~cm} \mathrm{x}$ $60 \mathrm{~cm}$ ) como preparo mínimo de solo. O plantio do povoamento decorreu no período de inverno do ano de 1999, portanto na ocasião do estudo o povoamento estava com 9 anos de idade. Foram utilizadas mudas clonais produzidas em tubetes no espaçamento de $3 \mathrm{~m} \times 2 \mathrm{~m}$. Aos 5 anos de idade todas as árvores sofreram poda (desrama) até 3,0 metros de altura.

\section{Inventário do povoamento}

O estudo foi realizado, em maio de 2008 , onde primeiramente foi realizado o inventário florestal, por meio da alocação de três parcelas amostrais $(30 \mathrm{~m} \times 20 \mathrm{~m})$. Nessas parcelas foram aferidos todos os diâmetros a altura do peito (DAP) e altura total (Tabela 1). O diâmetro foi aferido com uma fita diamétrica e a altura total com hipsômetro digital. O desbaste realizado foi do tipo sistemático, com a retirada da $9^{\text {a }}$ linha de plantio e seletivo, com a posterior retirada de indivíduos dominados pelo dossel do povoamento.

TABELA 1: Características dendrométricas do povoamento de Pinus taeda, antes e após o desbaste.

TABLE 1: Dentrometric characteristics of Pinus taeda stand, before and after the thinning.

\begin{tabular}{lccc}
\hline Variáveis & Atual & Remanescente & Desbastado \\
\hline $\mathrm{N}\left(\right.$ árvores ha $\left.{ }^{-1}\right)$ & 1565 & 1019 & 546 \\
DAP médio $(\mathrm{cm})$ & 21,3 & 22,1 & 19,6 \\
Altura média $(\mathrm{m})$ & 15,4 & 15,5 & 14,0 \\
Área Basal $\left(\mathrm{m}^{2} \mathrm{ha}^{-1}\right)$ & 55,6 & 38,8 & 16,8 \\
Volume $_{c / c}\left(\mathrm{~m}^{3} \mathrm{ha}^{-1}\right)$ & 376,5 & 271,1 & 105,4 \\
\hline
\end{tabular}


Após o inventário florestal, os indivíduos que foram retirados no desbaste, foram divididos em três classes diamétricas: 10,0 a $15,0 \mathrm{~cm} ; 15,1$ a $20,0 \mathrm{~cm}$ e 20,1 a $25,0 \mathrm{~cm}$, sendo seus respectivos centros de classe (CC): $12,5 \mathrm{~cm}, 17,5 \mathrm{~cm}$ e $22,5 \mathrm{~cm}$. A divisão nestas classes de diâmetros foi realizada conforme especificado, devido a variação dos mesmos no povoamento estudado ter sido de tal magnitude.

\section{Determinação da biomassa e dos nutrientes}

A determinação da biomassa baseouse na amostragem de 9 árvores, $3 \mathrm{em}$ cada classe diamétrica. Uma vez identificadas as árvores, as mesmas foram abatidas e separadas nos seguintes componentes: acículas vivas; acículas mortas; galhos vivos; galhos mortos; casca; madeira comercial e madeira do ponteiro com casca. Considerou-se ponteiro toda a parte do tronco a partir do ponto onde o diâmetro tornava-se inferior a oito centímetros.

Para a determinação do teor de umidade e concentração de nutrientes, coletaram-se amostras dos diferentes componentes. Para acícula, galho vivo e galho morto foi coletado uma amostra por árvore e componente. No caso da madeira e casca do fuste, foram três amostras por árvore e componente, nas seguintes posições: a 1,30 m (DAP), na metade do comprimento do fuste comercial e na extremidade superior, onde o diâmetro era igual a $8 \mathrm{~cm}$. Já para o ponteiro, coletou-se uma única amostra por árvore e componente, na região onde correspondia à metade do seu comprimento total. A quantificação da biomassa foi realizada com base na biomassa seca média de cada componente por classe de diâmetro e número de árvores por hectare na respectiva classe. A estimativa do estoque de nutrientes em cada componente das árvores foi possível mediante o produto do teor médio de nutrientes pela biomassa seca.

Após a coleta, as amostras foram secas em estufa de circulação e renovação de ar a $70^{\circ} \mathrm{C}$, por 72 horas, sendo posteriormente pesados em balança digital $(0,01 \mathrm{~g})$. Após as amostras foram moídas em moinho tipo Wiley com peneira de 30 mesh e posteriormente analisadas quanto aos teores de nitrogênio $(\mathrm{N})$, fósforo $(\mathrm{P})$, potássio $(\mathrm{K})$, cálcio $(\mathrm{Ca})$, magnésio $(\mathrm{Mg})$, enxofre $(\mathrm{S})$, boro $(\mathrm{B})$, cobre $(\mathrm{Cu})$, ferro $(\mathrm{Fe})$, manganês $(\mathrm{Mn})$ e zinco $(\mathrm{Zn})$. A determinação do $\mathrm{N}$ foi realizada por digestão sulfúrica $\left(\mathrm{H}_{2} \mathrm{SO}_{4}+\mathrm{H}_{2} \mathrm{O}_{2}\right)$ pelo método Kjeldahl. Para o $\mathrm{Ca}, \mathrm{Mg}, \mathrm{Cu}, \mathrm{Fe}, \mathrm{Mn}, \mathrm{Zn}$ pelo método de espectrometria de absorção atômica, $\mathrm{K}$ por fotometria de chama, $\mathrm{P}$ por espectrometria e $\mathrm{S}$ por turbidimetria, todos por digestão nítrica-perclórica $\left(\mathrm{HNO}_{3}+\mathrm{HClO}_{4}\right)$ [3:1], digestão seca pelo método de espectrometria para o boro), de acordo com a metodologia descrita por Tedesco et al. (1995) e Miyazawa et al., (1999).

As parcelas de inventário foram dispostas ao acaso, em três repetições. Foi realizado um teste de comparação de médias, Tukey a $5 \%$ de probabilidade de erro entre os nutrientes nas respectivas frações (fatores).

Para a simulação da quantidade de nutrientes a serem repostos no final do ciclo, em função da remoção destes através do primeiro desbaste, foram utilizados os adubos, no caso do fósforo optou-se pelo Super Fosfato Triplo (SPT) por ser mais concentrado que o Super Fosfato Simples (SPS), para o nitrogênio a ureia e para o potássio o cloreto de potássio. Para a obtenção da quantidade de adubos a serem repostos, realizou-se a multiplicação do fator de conversão para os óxidos (Tabela 2) com a quantidade de nutrientes a serem exportados, resultando nos nutrientes convertidos em óxidos. E finalmente para quantidade de adubos a serem repostos, multiplicou-se a porcentagem de nutrientes por adubo com os nutrientes convertidos em óxidos.

\section{RESULTADOS E DISCUSSÃO}

\section{Biomassa do desbaste}

A biomassa no primeiro desbaste foi de 44,5 $\mathrm{Mg} \mathrm{ha}^{-1}$, sendo composta, principalmente,

TABELA 2: Fatores de conversão em óxidos, para determinação da quantidade de nutrientes a serem repostos.

TABLE 2: Conversion factors in oxide to determine the quantity of nutrients to be reset.

\begin{tabular}{lcc}
\hline Elemento & Forma do Óxido & Fator \\
\hline Nitrogênio & $\mathrm{NO}_{3}{ }^{-}$ & 4,4268 \\
Fósforo & $\mathrm{P}_{2} \mathrm{O}_{5}$ & 2,29136 \\
Potássio & $\mathrm{K}_{2} \mathrm{O}^{-}$ & 1,20458 \\
\hline
\end{tabular}

Ci. Fl., v. 21, n. 3, jul.-set., 2011 
pelo compartimento madeira $(62,8 \%)$ (Tabela 3$)$. Schumacher et al. (2007) estudando também um povoamento de pinus em desbaste, na mesma região desse estudo, encontraram $35 \mathrm{Mg} \mathrm{ha}^{-1}$ de biomassa total, sendo $60,5 \%$ madeira comercial, aos 9 anos de idade.

Outros estudos, avaliando a biomassa total do povoamento, encontraram valores superiores, como por exemplo: Schumacher et al. (2002) estudando povoamentos de Pinus taeda com 10 anos de idade (950 árvores ha- ${ }^{-1}$ ), encontraram biomassa de 91,5 $\mathrm{Mg} \mathrm{ha}^{-1}$; Van Lear et al. (1984), encontraram para um povoamento de Pinus taeda, com 41 anos de idade, após dois desbastes, com uma densidade média de 437 árvores por hectare, uma biomassa de 109,6 $\mathrm{Mg} \mathrm{ha}^{-1}$; Castro et al. (1980), estudando Pinus oocarpa encontraram $102 \mathrm{Mg} \mathrm{ha}^{-1}$ aos 8 anos de idade do povoamento.

A distribuição dos componentes em ordem decrescente foi a seguinte: madeira $>$ galhos vivos $>$ casca $>$ acícula viva $>$ galho morto $>$ ponteiro $>$ acícula morta. Em povoamentos de Pinus oocarpa, com 8 anos de idade, Castro et al. (1980) observaram que a biomassa acima do solo era formada por $69,2 \%$ de madeira; $15,2 \%$ de casca; $9,9 \%$ de ramos e $5,7 \%$ de acículas.

Considerando que fosse explorada somente a madeira, juntamente com a casca, durante o primeiro desbaste, isso corresponderia a $71,7 \%$ da biomassa acima do solo. Sendo os restantes resíduos $(28,3 \%)$ que poderão ou não, devido às necessidades da empresa, ficar no sítio florestal. Segundo Valeri et al. (1989a), estudando a exportação de biomassa de povoamentos de $P$. taeda desbastados em diferentes idades, encontraram: para o primeiro desbaste, aos 7 anos de idade e com densidade de desbaste de $38,4 \%$ dos indivíduos, uma exportação (madeira + casca) de $11 \mathrm{Mg} \mathrm{ha}^{-1}$ e uma permanência de $7 \mathrm{Mg}$ ha $^{-1}$ de biomassa; já no segundo desbaste, aos 10 anos e com densidade de desbaste de $40,7 \%$, a exportação foi de $36 \mathrm{Mg}$ ha $^{-1}$ e a permanência de $10 \mathrm{Mg} \mathrm{ha}^{-1}$ de biomassa; e no terceiro desbaste, aos 14 anos e com densidade de desbaste de 39,3\%, a exportação atingiu $49 \mathrm{Mg}$ ha $^{-1}$ e uma permanência de $12 \mathrm{Mg} \mathrm{ha}^{-1}$ de biomassa acima do solo no povoamento. Para Schumacher et al. (2007) a biomassa total acumulada na ocasião do desbaste em um povoamento de Pinus taeda, aos 9 anos de idade foi de $35,68 \mathrm{Mg} \mathrm{ha}^{-1}$, sendo em $\mathrm{Mg}$ ha $^{-1}, 22,55$ de madeira; 4,21 de casca; 5,31 de galho e 3,61 de acículas.

\section{Nutrientes na biomassa \\ Teores de nutrientes na biomassa}

Os macronutrientes apresentaram maior concentração no componente acículas vivas, com exceção ao cálcio e magnésio, os quais foram superiores nas acículas mortas e galhos vivos, respectivamente (Tabela 4). Essa maior concentração nas acículas vivas deve-se a tendência de que a maioria dos nutrientes tem de concentrarse nas estruturas mais novas da planta, onde há uma maior atividade metabólica. Essa mesma tendência foi encontrada por La Torraca et al. (1984), em um povoamento de Pinus elliotii var. elliottii, por Bellote et al. (1980), em Eucalyptus grandis e por Pereira et al. (2000), em Acacia mearnsii.

Os diferentes componentes da biomassa apresentaram composições químicas estatisticamente distintas. Em relação ao teor de N,

TABELA 3: Biomassa a ser desbastada $\left(\mathrm{kg} \mathrm{ha}^{-1}\right)$ nos diferentes componentes das árvores do povoamento de Pinus taeda.

TABLE 3: Biomass to be thinning $\left(\mathrm{kg} \mathrm{ha}^{-1}\right)$ in different components the trees in Pinus taeda stand.

\begin{tabular}{|c|c|c|c|c|c|c|c|c|}
\hline \multirow{3}{*}{ Frações } & \multicolumn{6}{|c|}{ Classes diamétricas } & \multirow{2}{*}{\multicolumn{2}{|c|}{$\begin{array}{c}\text { Total de biomassa } \\
\text { desbastada }\end{array}$}} \\
\hline & \multicolumn{2}{|c|}{$10,0 \mid-15,0$} & \multicolumn{2}{|c|}{$15,1 \mid-20,0$} & \multicolumn{2}{|c|}{$20,1 \mid-25,0$} & & \\
\hline & $\mathrm{kg} \mathrm{ha}^{-1}$ & $\%$ & $\mathrm{~kg} \mathrm{ha}^{-1}$ & $\%$ & $\mathrm{~kg} \mathrm{ha}^{-1}$ & $\%$ & $\mathrm{~kg} \mathrm{ha}^{-1}$ & $\%$ \\
\hline $\mathrm{C}$ & 561,1 & 18,6 & $1.152,7$ & 12,1 & $2.230,0$ & 7,0 & $3.943,8$ & 8,9 \\
\hline $\mathrm{AM}$ & 19,9 & 0,7 & 110,7 & 1,2 & 93,4 & 0,3 & 224,0 & 0,5 \\
\hline A V & 219,4 & 7,3 & 770,5 & 8,1 & $2.512,5$ & 7,9 & $3.502,4$ & 7,9 \\
\hline G M & 210,4 & 7,0 & 816,2 & 8,6 & $1.703,0$ & 5,3 & $2.729,6$ & 6,1 \\
\hline G V & 173,6 & 5,8 & 864,6 & 9,1 & $3.550,0$ & 11,1 & $4.588,2$ & 10,3 \\
\hline M & $1.459,2$ & 48,4 & $5.322,2$ & 55,8 & $21.127,7$ & 66,2 & $27.909,2$ & 62,8 \\
\hline $\mathrm{P}$ & 371,1 & 12,3 & 507,1 & 5,3 & 697,0 & 2,2 & $1.575,2$ & 3,5 \\
\hline Total & $3.014,7$ & 100,0 & $9.544,0$ & 100,0 & $31.913,6$ & 100,0 & $44.472,3$ & 100,0 \\
\hline
\end{tabular}

Em que: $\mathrm{C}=$ casca; $\mathrm{AM}=$ Acícula Morta; $\mathrm{AV}=$ Acícula Viva; GM = Galho Morto; GV = Galho Vivo; $\mathrm{M}=\mathrm{Madeira}$; $\mathrm{P}=$ Ponteiro. $\%=\mathrm{O}$ percentual de cada fração em relação à biomassa desbastada. 
TABELA 4: Teores de macronutrientes $\left(\mathrm{g} \mathrm{kg}^{-1}\right)$ nos componentes na biomassa de Pinus taeda.

TABLE 4: Levels of macronutrients $\left(\mathrm{g} \mathrm{kg}^{-1}\right)$ in components in the biomass of Pinus taeda.

\begin{tabular}{ccccccc}
\hline \multirow{2}{*}{ Frações } & $\mathrm{N}$ & $\mathrm{P}$ & $\mathrm{K}$ & $\mathrm{Ca}$ & $\mathrm{Mg}$ & $\mathrm{S}$ \\
\cline { 2 - 7 } & \multicolumn{7}{c}{$\mathrm{g} \mathrm{kg}^{-1}$} & $0,84 \mathrm{~b}$ & $0,99 \mathrm{e}$ & $0,31 \mathrm{~b}$ & $0,53 \mathrm{bc}$ \\
$\mathrm{C}$ & $2,84 \mathrm{de}$ & $0,28 \mathrm{de}$ & $0,84 \mathrm{~b}$ & $5,50 \mathrm{a}$ & $0,87 \mathrm{ab}$ & $0,81 \mathrm{a}$ \\
$\mathrm{A} \mathrm{M}$ & $6,98 \mathrm{~b}$ & $0,45 \mathrm{~cd}$ & $1,23 \mathrm{~b}$ & $3,52 \mathrm{bc}$ & $0,96 \mathrm{a}$ & $0,86 \mathrm{a}$ \\
$\mathrm{A} \mathrm{V}$ & $13,85 \mathrm{a}$ & $1,21 \mathrm{a}$ & $7,43 \mathrm{a}$ & $3,91 \mathrm{~b}$ & $0,87 \mathrm{ab}$ & $0,55 \mathrm{bc}$ \\
$\mathrm{G} \mathrm{M}$ & $3,31 \mathrm{cde}$ & $0,28 \mathrm{de}$ & $0,51 \mathrm{~b}$ & $3,47 \mathrm{~cd}$ & $1,01 \mathrm{a}$ & $0,59 \mathrm{~b}$ \\
$\mathrm{G} \mathrm{V}$ & $4,83 \mathrm{c}$ & $0,65 \mathrm{~b}$ & $1,89 \mathrm{~b}$ & 2,47 & $0,45 \mathrm{c}$ \\
M & $1,76 \mathrm{e}$ & $0,21 \mathrm{e}$ & $0,74 \mathrm{~b}$ & $0,94 \mathrm{e}$ & $0,31 \mathrm{~b}$ & $0,40 \mathrm{c}$ \\
P & $3,87 \mathrm{~cd}$ & $0,52 \mathrm{bc}$ & $1,93 \mathrm{~b}$ & $1,51 \mathrm{de}$ & $0,57 \mathrm{ab}$ & $0,60 \mathrm{~b}$ \\
\hline
\end{tabular}

Em que: $\mathrm{C}=$ casca; $\mathrm{AM}=$ Acícula Morta; $\mathrm{AV}=$ Acícula Viva; $\mathrm{GM}=$ Galho Morto; $\mathrm{GV}=$ Galho Vivo; $\mathrm{M}=\mathrm{Madeira}$; $\mathrm{P}=$ Ponteiro. Médias seguidas pela mesma letra, na vertical, não diferem entre si pelo Teste de Tukey a $5 \%$ de probabilidade de erro.

ocorre um domínio no componente acículas vivas, tendo um teor superior que nos demais componentes. $\mathrm{O}$ menor teor de nitrogênio foi encontrado na madeira comercial, que não diferiu dos galhos mortos e da casca. Esse teor de N, bem superior nas acículas que nos demais componentes, é devido a este elemento participar da maioria das reações de metabolismo de compostos estruturais como aminoácidos, proteínas, aminas, amidas, vitaminas, bases nitrogenadas e na participação de processos metabólicos como absorção iônica, fotossíntese, respiração, multiplicação e diferenciação celular, os quais têm a principal ocorrência nas acículas vivas (FERRI, 1985; MARENCO E LOPES, 2005; GRASSI FILHO, 2009).

Os teores de $\mathrm{P}$ apresentaram comportamento semelhante ao do $\mathrm{N}$, possuindo o maior teor nas acículas vivas e os menores teores na madeira, casca e galhos mortos, que não diferiram estatisticamente. A exemplo do nitrogênio, segundo Brun (2004), o fósforo tem ampla mobilidade dentro da planta, e com isso ele tende a se concentrar nos órgãos mais novos, no caso deste estudo no componente acículas. Para o Ko maior teor foi nas acículas vivas, diferindo estatisticamente dos demais componentes. Já para as demais frações não houve diferença estatística.

O cálcio apresentou seus maiores teores nas acículas mortas, sendo superior estatisticamente. Já nas demais frações madeira, casca e o ponteiro, apresentaram os menores teores desse elemento. $\mathrm{O}$ cálcio é um elemento estrutural, faz parte da parede celular, é tido como imóvel, por isso, apresenta a tendência de elevação dos teores com a senescência dos tecidos, que é o caso das acículas mortas e os galhos mortos. Nas acículas vivas e galhos vivos apresentaram os maiores teores de $\mathrm{Mg}$, sendo superiores as demais frações. $\mathrm{O}$ enxofre demonstrou a mesma tendência do $\mathrm{N}$ e o P sendo superiores nas acículas vivas e inferiores na madeira.

O boro está mais concentrado nas acículas vivas e acículas mortas e os menores na madeira (Tabela 5), isto porque o boro atua no crescimento meristemático, metabolismo da membrana celular e também dos carboidratos (GRASSI FILHO, 2009). $\mathrm{O}$ componente galhos vivos foi o que apresentou o maior teor de cobre. As acículas mortas apresentaram os menores teores de cobre não diferindo sua concentração dos demais componentes, sendo este elemento o menor exigido por esta espécie (CHAVES e CORREAA, 2003). O menor teor de ferro está presente na casca e os maiores valores foram encontrados nas acículas mortas. Os teores de zinco foram estatisticamente superiores para a casca da ponteira e para as acículas e inferiores para a madeira comercial. Em termos gerais os componentes com os maiores teores de nutrientes foram as acículas vivas e mortas, que só não apresentaram teor mais alto no cobre. E o componente com menor teor de micronutrientes foi a madeira acícula viva e para os galhos, tanto vivos quanto mortos. Segundo McGrath e Robson (1984) apud Martinez et al., (1992), o processo de concentração dos teores de $\mathrm{Zn}$ nos ramos de pinus parece ser um processo físicoquímico, não metabólico, que ocorre em situações de provisão escassa de Zn no solo.

\section{Quantidade de nutrientes na biomassa}

A quantidade de nitrogênio acumulada na biomassa resultante do primeiro desbaste foi a maior dentre todos os nutrientes (Tabela 6). O maior acúmulo de $\mathrm{N}$ foi encontrado na madeira, apesar de ter menor teor, mas em compensação possui a maior biomassa entre os componentes. A madeira e os galhos vivos possuem as maiores quantidades 
TABELA 5: Teores de micronutrientes $\left(\mathrm{mg} \mathrm{kg}^{-1}\right)$ nos componentes na biomassa de Pinus taeda. TABLE 5: Levels of micronutrients $\left(\mathrm{mg} \mathrm{kg}^{-1}\right)$ in components in the biomass of Pinus taeda.

\begin{tabular}{cccccc}
\hline \multirow{2}{*}{ Frações } & $\mathrm{B}$ & $\mathrm{Cu}$ & $\mathrm{Fe}$ & $\mathrm{Mn}$ & $\mathrm{Zn}$ \\
\cline { 2 - 6 } & \multicolumn{5}{c}{$\mathrm{mg} \mathrm{kg}^{-1}$} \\
$\mathrm{C}$ & $8,39 \mathrm{bc}$ & $5,58 \mathrm{ab}$ & $96,28 \mathrm{bc}$ & $75,48 \mathrm{~d}$ & $11,30 \mathrm{~cd}$ \\
$\mathrm{~A} \mathrm{M}$ & $11,57 \mathrm{a}$ & $1,31 \mathrm{~d}$ & $196,28 \mathrm{a}$ & $1.062,68 \mathrm{a}$ & $25,63 \mathrm{ab}$ \\
$\mathrm{A} \mathrm{V}$ & $11,92 \mathrm{a}$ & $2,08 \mathrm{~cd}$ & $59,59 \mathrm{cde}$ & $625,20 \mathrm{~b}$ & $30,18 \mathrm{a}$ \\
$\mathrm{G} \mathrm{M}$ & $9,63 \mathrm{ab}$ & $5,81 \mathrm{a}$ & $136,44 \mathrm{~b}$ & $332,97 \mathrm{c}$ & $20,26 \mathrm{abc}$ \\
G V & $9,39 \mathrm{ab}$ & $7,25 \mathrm{a}$ & $81,44 \mathrm{~cd}$ & $212,96 \mathrm{~cd}$ & $22,52 \mathrm{ab}$ \\
M & $2,99 \mathrm{~d}$ & $3,83 \mathrm{ab}$ & $17,78 \mathrm{e}$ & $130,77 \mathrm{~cd}$ & $6,90 \mathrm{~d}$ \\
P & $6,65 \mathrm{c}$ & $6,00 \mathrm{a}$ & $33,83 \mathrm{de}$ & $127,04 \mathrm{~cd}$ & $17,64 \mathrm{bcd}$ \\
\hline
\end{tabular}

Em que: $\mathrm{C}=$ casca; $\mathrm{AM}=$ Acícula Morta; $\mathrm{AV}=$ Acícula Viva; $\mathrm{GM}=$ Galho Morto; $\mathrm{GV}=$ Galho Vivo; $\mathrm{M}=\mathrm{Madeira}$; $\mathrm{P}=$ Ponteiro. Médias seguidas pela mesma letra, na vertical, não diferem entre si pelo Teste de Tukey a 5\% de probabilidade de erro.

TABELA 6: Quantidade de macronutrientes $\left(\mathrm{kg} \mathrm{ha}^{-1}\right)$ nos componentes na biomassa de Pinus taeda. TABLE 6: Macronutrients quantity $\left(\mathrm{kg} \mathrm{ha}^{-1}\right)$ in the biomass components of Pinus taeda.

\begin{tabular}{cccccccccccccc}
\hline \multirow{2}{*}{ Frações } & \multicolumn{2}{c}{$\mathrm{N}$} & \multicolumn{2}{c}{$\mathrm{P}$} & \multicolumn{2}{c}{$\mathrm{K}$} & \multicolumn{2}{c}{$\mathrm{Ca}$} & \multicolumn{2}{c}{$\mathrm{Mg}$} & \multicolumn{2}{c}{$\mathrm{S}$} \\
\cline { 2 - 14 } $\mathrm{kg} \mathrm{ha}^{-1}$ & $\%$ & $\mathrm{~kg} \mathrm{ha}^{-1}$ & $\%$ & $\mathrm{~kg} \mathrm{ha}^{-1}$ & $\%$ & $\mathrm{~kg} \mathrm{ha}^{-1}$ & $\%$ & $\mathrm{~kg} \mathrm{~h}^{-1}$ & $\%$ & $\mathrm{~kg} \mathrm{ha}^{-1}$ & $\%$ \\
\hline C & 11,46 & 11,40 & 1,10 & 9,39 & 3,62 & 7,74 & 3,66 & 7,17 & 1,22 & 8,50 & 2,01 & 11,02 \\
A M & 1,50 & 1,49 & 0,10 & 0,87 & 0,20 & 0,43 & 1,27 & 2,48 & 0,19 & 1,33 & 0,18 & 0,99 \\
A V & 5,75 & 5,72 & 0,51 & 4,33 & 3,24 & 6,93 & 1,43 & 2,80 & 0,41 & 2,85 & 0,37 & 2,01 \\
G M & 8,77 & 8,73 & 0,72 & 6,12 & 1,23 & 2,63 & 10,40 & 20,35 & 1,79 & 12,51 & 1,41 & 7,75 \\
G V & 25,28 & 25,15 & 3,40 & 29,07 & 12,81 & 27,38 & 8,81 & 17,22 & 3,34 & 23,34 & 2,77 & 15,23 \\
M & 41,47 & 41,26 & 5,05 & 43,14 & 22,40 & 47,87 & 23,30 & 45,57 & 6,51 & 45,51 & 10,55 & 57,88 \\
P & 6,28 & 6,25 & 0,83 & 7,08 & 3,28 & 7,01 & 2,25 & 4,41 & 0,85 & 5,96 & 0,93 & 5,12 \\
\hline Total & 100,51 & 100,00 & 11,70 & 100,00 & 46,79 & 100,00 & 51,12 & 100,00 & 14,31 & 100,00 & 18,22 & 100,00 \\
\hline
\end{tabular}

Em que: $\mathrm{C}=$ casca; $\mathrm{AM}=$ Acícula Morta; $\mathrm{AV}=$ Acícula Viva; $\mathrm{GM}=$ Galho Morto; $\mathrm{GV}=$ Galho Vivo; $\mathrm{M}=\mathrm{Madeira;}$ $\mathrm{P}=$ Ponteiro.

de fósforo e potássio, ultrapassando $72 \%$ do total presente na biomassa. Os maiores estoques de cálcio, magnésio e enxofre estão contidos na madeira comercial. Sendo que, esse componente possui mais de $58 \%$ do S, presente na biomassa total.

A madeira e os galhos vivos são as frações que mais armazenam nutrientes entre os componentes da biomassa em função da maior alocação de biomassa. A importância em armazenamento para os macronutrientes nos componentes da biomassa segue a seguinte ordem: madeira $>$ galhos vivos $>$ galhos mortos $>$ casca $>$ ponteiro $>$ acícula viva $>$ acícula morta. Quanto à magnitude de armazenamento dos elementos, a ordem foi a seguinte: $\mathrm{N}>\mathrm{Ca}>\mathrm{K}>\mathrm{S}>\mathrm{Mg}>\mathrm{P}$.

Entretanto, para Pinus oocarpa, aos 8 anos, Castro et al. (1980), e em Acacia mearnsii, aos 9 anos, Pereira et al. (2000), encontraram a seguinte magnitude de armazenamento: $\mathrm{N}>\mathrm{K}>\mathrm{Ca}>\mathrm{Mg}>$ P e em Eucalyptus grandis, aos 7 anos, Bellote et al.
(1980), obtiveram: $\mathrm{Ca}>\mathrm{N}>\mathrm{K}>\mathrm{S}>\mathrm{Mg}>\mathrm{P}$.

No estudo realizado por Valeri et al. (1989b), os resíduos (acículas, galhos, ponteiro e casca) foram responsáveis por cerca de: $76 \%$ do N, $68 \%$ do P, $68 \%$ do $\mathrm{K}, 66 \%$ do $\mathrm{Mg}$ e $57 \%$ do Ca, no primeiro desbaste quando o povoamento encontrava-se com 7 anos de idade; $60,2 \%$ do N, $50 \%$ do P, $48 \%$ do $\mathrm{K}, 48 \%$ do $\mathrm{Mg}$ e $35 \%$ do $\mathrm{Ca}$, no segundo desbaste aos 10 anos de idade; $58 \%$ do N, $49 \%$ do P, $46 \%$ do $\mathrm{Mg}, 43 \%$ do $\mathrm{K}$ e $35 \%$ do $\mathrm{Ca}$, no terceiro desbaste aos 14 anos de idade. Fazendo-se esta opção, pela permanência dos resíduos (ponteiro, galhos vivos e mortos e acículas vivas e mortas) no interior do povoamento, cerca de $59 \%$ do N, $57 \%$ do P, $55 \%$ do $\mathrm{Ca}$ e $\mathrm{Mg}$, e $53 \%$ do $\mathrm{K}$, do total presente nas árvores desbastadas permanecerão no sítio florestal. As proporções encontradas estão de acordo com o segundo desbaste, aos 10 anos, do autor acima citado, pois neste trabalho o primeiro desbaste foi realizado aos 9 anos. 
Em povoamentos de Pinus oocarpa, com 8 anos de idade, a exploração apenas da madeira comercial reduziria a exportação de $\mathrm{N}$ em $48 \%$, P em $40 \%$, K em $38 \%$, Ca em $29 \%$ e Mg em 39\%, e com a permanência da casca no sítio florestal, com remoção somente da madeira, a exportação do nitrogênio seria reduzida em $65 \%$, fósforo em $54 \%$, cálcio em $53 \%$ e potássio e magnésio em $52 \%$ (CASTRO et al., 1980). Estes valores apresentados pelo autor citado, anteriormente, vão de encontro aos encontrados no estudo, entretanto somente o valor de nitrogênio, que tem a maior diferença.

Em relação aos micronutrientes, o manganês foi o que apresentou a maior quantidade, com maior acúmulo nos galhos mortos e na madeira (Tabela 7), a qual também obteve os maiores acúmulos dos demais micronutrientes.

A importância em armazenamento para os micronutrientes nos componentes da biomassa segue a seguinte magnitude: madeira $>$ galhos mortos $>$ galhos vivos $>$ acícula viva $>$ casca $>$ ponteiro $>$ acícula morta. A ordem decrescente de micronutrientes acumulados foi: $\mathrm{Mn}, \mathrm{Fe}$, $\mathrm{Zn}, \mathrm{B}$ e $\mathrm{Cu}$. Com a permanência dos resíduos (acículas, galhos, ponteiro e casca) no interior do povoamento, cerca de $59 \%$ do Fe, $58 \%$ do $\mathrm{Zn}, 55 \%$ do $\mathrm{B}, 42 \%$ do $\mathrm{Cu}$ e $41 \%$ do $\mathrm{Mn}$, do total presente nas árvores desbastadas, irão retornar ao solo após a decomposição desse material, estando disponível para as árvores que permaneceram no povoamento. Entretanto, no estudo realizado por Valeri et al. (1989b), citado anteriormente, os resíduos (acículas, galhos, ponteiro e casca) foram responsáveis por cerca de: $70 \%$ do $\mathrm{Mn}, 58 \%$ do $\mathrm{Zn}, 54 \%$ do Fe, $52 \%$ do $\mathrm{Cu}$ e $51 \%$ do $\mathrm{B}$, no primeiro desbaste; $51 \%$ do $\mathrm{Mn}, 36 \%$ do $\mathrm{Zn}, 36 \%$ do $\mathrm{Cu}, 30 \%$ do $\mathrm{B}$ e $29 \%$ do
Fe, no segundo desbaste e; $52 \%$ do $\mathrm{Mn}, 32 \%$ do $\mathrm{Zn}$, $29 \%$ do $\mathrm{B}, 27 \%$ do $\mathrm{Fe}$ e $25 \%$ do $\mathrm{Cu}$, no terceiro desbaste.

\section{Exportação de nutrientes}

O sistema de exploração mais utilizado pelas empresas é a retirada da madeira juntamente com a sua casca, sendo esta, na indústria, utilizada como material energético nas caldeiras. Devido à necessidade de utilização da casca da madeira, na indústria, o sistema de exploração: madeira + casca, até um diâmetro de $8 \mathrm{~cm}$, seria o mais indicado. Pois, além de haver biomassa para a combustão (casca), esse sistema de exploração possibilita um grande volume de resíduos sobre o solo, os quais disponibilizariam uma grande quantidade de nutrientes após a decomposição do resíduo. Sendo, desta maneira, exportado com o referido sistema, $68,9 \%$ de $\mathrm{S} ; 55,6 \%$ de $\mathrm{K} ; 54,0 \%$ de $\mathrm{Mg} ; 52,7 \%$ de $\mathrm{N}$ e $52,5 \%$ de $\mathrm{P}$ (Tabela 8), dos macronutrientes presentes na biomassa total oriunda do primeiro desbaste.

Nos micronutrientes a exportação foi de: $64,2 \%$ de $\mathrm{Cu} ; 61,3 \%$ de $\mathrm{Mn} ; 52,8 \%$ de $\mathrm{B} ; 51,9 \%$ de $\mathrm{Fe}$ e $47,0 \%$ de $\mathrm{Zn}$ (Tabela 9). A variação percentual de cada sistema de exploração florestal em relação aos diferentes elementos minerais presentes na biomassa. Pode-se notar que há uma pequena diferença, na exportação de nutrientes, entre a exploração da madeira + casca e a exploração da madeira + casca + ponteiro, até os $8 \mathrm{~cm}$ de diâmetro do fuste. Deve-se a isso a pequena quantidade de biomassa que apresenta o ponteiro (ponta da copa, com diâmetro de fuste inferior a $8 \mathrm{~cm}$ ), mesmo ele contendo teores altos dos diferentes elementos.

TABELA 7: Quantidade de micronutrientes $\left(\mathrm{g} \mathrm{ha}^{-1}\right)$ nos componentes na biomassa de Pinus taeda. TABLE 7: Micronutrients quantity $\left(\mathrm{g} \mathrm{ha}^{-1}\right)$ in Pinus taeda biomass components.

\begin{tabular}{ccccccccccc}
\hline \multirow{2}{*}{ Frações } & \multicolumn{3}{c}{$\mathrm{B}$} & \multicolumn{3}{c}{$\mathrm{Cu}$} & \multicolumn{2}{c}{$\mathrm{Fe}$} & \multicolumn{2}{c}{$\mathrm{Mn}$} \\
\cline { 2 - 11 } & $\mathrm{g} \mathrm{ha}^{-1}$ & $\%$ & $\mathrm{~g} \mathrm{ha}^{-1}$ & $\%$ & $\mathrm{~g} \mathrm{ha}^{-1}$ & $\%$ & $\mathrm{~g} \mathrm{ha}^{-1}$ & $\%$ & $\mathrm{~g} \mathrm{ha}^{-1}$ & $\%$ \\
\hline C & 14,12 & 7,79 & 9,39 & 5,28 & 162,07 & 10,20 & 127,06 & 2,12 & 19,03 & 4,37 \\
A M & 2,65 & 1,46 & 0,32 & 0,18 & 45,40 & 2,86 & 226,74 & 3,78 & 5,30 & 1,22 \\
A V & 5,03 & 2,78 & 0,87 & 0,49 & 25,00 & 1,57 & 290,28 & 4,84 & 13,37 & 3,07 \\
G M & 25,28 & 13,95 & 15,26 & 8,59 & 379,43 & 23,89 & 902,73 & 15,06 & 60,00 & 13,79 \\
G V & 42,15 & 23,26 & 37,43 & 21,06 & 261,27 & 16,45 & 706,21 & 11,78 & 123,66 & 28,43 \\
M & 81,62 & 45,05 & 104,73 & 58,93 & 662,45 & 41,70 & $3.546,04$ & 59,17 & 185,33 & 42,61 \\
P & 10,33 & 5,70 & 9,71 & 5,46 & 52,92 & 3,33 & 193,53 & 3,23 & 28,29 & 6,50 \\
\hline Total & 181,18 & 100,00 & 177,71 & 100,00 & $1.588,55$ & 100,00 & $5.992,59$ & 100,00 & 434,98 & 100,00 \\
\hline
\end{tabular}

Em que: $\mathrm{C}=$ casca; $\mathrm{AM}=$ Acícula Morta; $\mathrm{AV}=$ Acícula Viva; $\mathrm{GM}=$ Galho Morto; $\mathrm{GV}=$ Galho Vivo; $\mathrm{M}=\mathrm{Madeira}$; $\mathrm{P}=$ Ponteiro.

Ci. Fl., v. 21, n. 3, jul.-set., 2011 
TABELA 8: Quantidades de macronutrientes $\left(\mathrm{kg} \mathrm{ha}^{-1}\right)$ exportados através de diferentes intensidades de utilização da biomassa do primeiro desbaste.

TABLE 8: Macronutrients quantities $\left(\mathrm{kg} \mathrm{ha}^{-1}\right)$ exported by different intensities of biomass usage in the first thinning.

\begin{tabular}{lcccccc}
\hline Exploração & $\mathrm{N}$ & $\mathrm{P}$ & $\mathrm{K}$ & $\mathrm{Ca}$ & $\mathrm{Mg}$ & $\mathrm{S}$ \\
\hline \multirow{2}{*}{ Total } & 100,5 & 11,7 & 46,8 & 51,1 & 14,3 & 18,2 \\
& $(100,0)$ & $(100,0)$ & $(100,0)$ & $(100,0)$ & $(100,0)$ & $(100,0)$ \\
$\mathrm{M}+\mathrm{C}+\mathrm{P}$ & 59,2 & 7,0 & 29,3 & 29,2 & 8,6 & 13,5 \\
& $(58,9)$ & $(59,6)$ & $(62,6)$ & $(57,1)$ & $(60,0)$ & $(74,0)$ \\
$\mathrm{M}+\mathrm{C}$ & 52,9 & 6,1 & 26,0 & 27,0 & 7,7 & 12,6 \\
& $(52,7)$ & $(52,5)$ & $(55,6)$ & $(52,7)$ & $(54,0)$ & $(68,9)$ \\
$\mathrm{M}$ & 41,5 & 5,0 & 22,4 & 23,3 & 6,5 & 10,5 \\
& $(41,3)$ & $(43,1)$ & $(47,9)$ & $(45,6)$ & $(45,5)$ & $(57,9)$ \\
\hline
\end{tabular}

Em que: $\mathrm{C}=$ Casca; $\mathrm{M}=$ Madeira; $\mathrm{P}=$ Ponteiro. Valores entre parênteses, referem-se à porcentagem de macronutrientes exportados em cada sistema de exploração em relação ao total presente na biomassa.

TABELA 9: Quantidades de micronutrientes $\left(\mathrm{g} \mathrm{ha}^{-1}\right)$ exportados através de diferentes intensidades de utilização da biomassa do primeiro desbaste.

TABLE 9: Micronutrients quantities $\left(\mathrm{kg} \mathrm{ha}^{-1}\right)$ exported by different intensities of biomass usage in the first thinning.

\begin{tabular}{lccccc}
\hline Exploração & $\mathrm{B}$ & $\mathrm{Cu}$ & $\mathrm{Fe}$ & $\mathrm{Mn}$ & $\mathrm{Zn}$ \\
\hline \multirow{2}{*}{ Total } & 181,2 & 177,7 & $1.588,6$ & $5.992,6$ & 435,0 \\
& $(100,0)$ & $(100,0)$ & $(100,0)$ & $(100,0)$ & $(100,0)$ \\
$\mathrm{M}+\mathrm{C}+\mathrm{P}$ & 106,1 & 123,8 & 877,4 & $3.866,6$ & 232,6 \\
& $(58,5)$ & $(69,7)$ & $(55,2)$ & $(64,5)$ & $(53,5)$ \\
$\mathrm{M}+\mathrm{C}$ & 95,7 & 114,1 & 824,5 & $3.673,1$ & 204,4 \\
& $(52,8)$ & $(64,2)$ & $(51,9)$ & $(61,3)$ & $(47,0)$ \\
$\mathrm{M}$ & 81,6 & 104,7 & 662,5 & $3.546,0$ & 185,3 \\
& $(45,0)$ & $(58,9)$ & $(41,7)$ & $(59,2)$ & $(42,6)$ \\
\hline
\end{tabular}

Em que: $\mathrm{C}=$ Casca; $\mathrm{M}=$ Madeira; $\mathrm{P}=$ Ponteiro. Valores entre parênteses, referem-se à porcentagem de micronutrientes exportados em cada sistema de exploração em relação ao total presente na biomassa.

Verifica-se também, que a alternativa mais correta, ao nível de sustentabilidade do sistema de produção em estudo, em relação à nutrição florestal, é apenas a retirada da madeira comercial. Mas como normalmente isso não é possível, devido à necessidade de utilização da casca e a difícil separação da madeira, faz-se necessário que sejam aplicados fertilizantes (adubos químicos ou orgânicos), que pelo menos supram em parte a quantidade de nutrientes retirados do solo e exportados para fora do sítio local após a colheita. Cabe ressaltar, ainda que, se essa adição de nutrientes não for feita, possivelmente a produtividade dos povoamentos, nas rotações futuras, poderá ser menor, aquém da satisfatória para a necessidade da empresa.

\section{Reposição nutricional}

A quantidade a ser reposta de nutrientes para os diferentes tipos de exploração está na Tabela
10. Nota-se que quanto maior for a exploração da biomassa maior terá que ser a reposição dos nutrientes através da adubação mineral. Esta reposição deverá ser realizada para que haja uma manutenção da produtividade em futuras rotações.

A quantidade de adubos a serem repostos vai depender do tipo de exploração da biomassa florestal adotado, se essa for a remoção total da biomassa certamente a quantidade de adubos a ser reposto será muito alta e onerosa, já considerando apenas a retirada da madeira esta reposição será aproximadamente $60 \%$ inferior do que na remoção total.

Também temos que levar em consideração que a reposição nutricional através da fertilização via adubação química em grandes quantidades pode ocasionar a perda destes nutrientes, por a planta não ter a capacidade de absorver uma grande quantidade de nutrientes e este excesso também pode acarretar na contaminação dos recursos hídricos. 
TABELA 10: Simulação da quantidade de adubos a serem repostos para os diferentes tipos de exploração do primeiro desbaste.

TABLE 10: Simulation of the amount of fertilizer to be reset for the different types of logging in the first thinning.

\begin{tabular}{lcccc}
\hline \multirow{2}{*}{ Exploração florestal } & Ureia & SPT & Cloreto de Potássio & Total \\
\cline { 2 - 5 } & & \multicolumn{3}{c}{$\mathrm{kg} \mathrm{ha}^{-1}$} \\
\hline Biomassa Total & 228,4 & 65,4 & 97,2 & 391,0 \\
M + C + P & 134,6 & 39,0 & 60,8 & 234,4 \\
M + C & 120,3 & 34,4 & 54,0 & 208,7 \\
M & 94,2 & 28,2 & 46,5 & 169,0 \\
\hline
\end{tabular}

Em que: $\mathrm{C}=$ Casca; $\mathrm{M}=$ Madeira; $\mathrm{P}=$ Ponteiro. Composição dos adubos: Ureia 44\% de N, SPT 41\% de $\mathrm{P}_{2} \mathrm{O}_{5}$ e Cloreto de potássio $58 \%$ de $\mathrm{K}_{2} \mathrm{O}$.

Para que haja sustentabilidade florestal e sucessivos ciclos, deverá haver a reposição dos nutrientes, para que não haja o esgotamento destes no solo e por consequência a diminuição da produção florestal.

\section{CONCLUSÃO}

A biomassa totalizou $44,5 \mathrm{Mg} \mathrm{ha}^{-1}$, com $62,8 \%$ de madeira, $10,3 \%$ de galhos vivos, $8,9 \%$ de casca, 10,1\% de acículas viva, 6,1\% de galho morto, $3,5 \%$ de ponteira e $0,5 \%$ de acícula morta.

A quantidade total de macronutrientes contidos na biomassa, em $\mathrm{kg} \mathrm{ha}^{-1}$, foi de: 100,5 de $\mathrm{N} ; 51,1$ de $\mathrm{Ca} ; 46,8$ de $\mathrm{K} ; 18,2$ de $\mathrm{S} ; 14,3$ de $\mathrm{Mg}$ e 11,7 de $\mathrm{P}$, para os micronutrientes, em $\mathrm{g} \mathrm{ha}^{-1}$, 5.995,6 de Mn; 1.588,6 de Fe; 435,0 de Zn; 181,2 de $\mathrm{B}$ e 177,7 de $\mathrm{Cu}$.

A quantidade de adubos a serem repostos vai depender do tipo da exploração, se for a remoção total a quantidade de adubos a ser reposto será de $391 \mathrm{~kg} \mathrm{ha}^{-1}$, já considerando apenas a retirada da madeira esta reposição será aproximadamente $60 \%$ inferior, $169 \mathrm{~kg} \mathrm{ha}^{-1}$.

A remoção da biomassa de pinus, durante o primeiro desbaste, deve envolver somente o componente madeira comercial, já que incluindo os demais componentes ocasionará uma elevada exportação de nutrientes e consequentemente uma elevada reposição de nutrientes.

\section{AGRADECIMENTOS}

Os autores agradecem a empresa Cambará S.A. pelos recursos disponibilizados para a realização do trabalho e pelo apoio logístico.

\section{REFERÊNCIAS BIBLIOGRÁFICAS}

BARICHELLO, L. R. Quantificação da biomassa e dos nutrientes em floresta de Acacia mearnsii De Wild. na região sul do Brasil. 2003. 58 f. Dissertação (Mestrado em Engenharia Florestal)Universidade Federal de Santa Maria, Santa Maria, 2003.

BARROS FILHO, N. F. Produção e participação de biomassa e de nutrientes e recomendação de fertilizantes para o Pinus taeda L. (Nutripinus). 2003. 56 f. Dissertação (Mestrado em Engenharia Florestal)-Universidade Federal de Santa Maria, Santa Maria, 2003.

BELLOTE, A. F. J. et al. Extração e exportação de nutriente pelo Eucalyptus grandis Hill ex-Maiden em função da idade: 1 - macronutrientes. IPEF, Piracicaba: n. 20, p. 27-45, 1980

BRUN, E. J. Biomassa e nutrientes na Floresta Estacional Decidual, em Santa Tereza, RS. 2004. 152 f. Dissertação (Mestrado em Engenharia Florestal)-Universidade Federal de Santa Maria, Santa Maria, 2004.

CASTRO, C. F. A. et al. Distribuição da fitomassa e nutrientes em talhões de Pinus oocarpa com diferentes idades. IPEF, Piracicaba: n. 20, p. 61-70, jun. 1980.

CHAVES, R. Q.; CORRÊA, G. F. Micronutrientes no sistema solo-Pinus caribea Morelet em palntios apresentando amarelecimento das acículas e morte de plantas. Revista Árvore, Viçosa, v. 27, n. 6, p. 769 - 778, 2003.

FERREIRA, C. A. et al. Nutrição de pinus no sul do Brasil: diagnóstico e propriedades de pesquisa. Documentos EMBRAPA Florestas, Colombo, n. 60, 2001. p. 1-23. 
FERRI, M. G. Fisiologia vegetal 1. São Paulo: EPU, 1985. p. 77-116.

FREITAS, R. A. Estudo da biomassa e do conteúdo de nutrientes em um povoamento de Eucalyptus grandis Hill ex Maiden plantado em solo sujeito a arenização, no município de Alegrete - RS. 2000. 60 f. Dissertação (Mestrado em Engenharia Florestal)-Universidade Federal de Santa Maria, Santa Maria, 2000.

GRASSI FILHO, H. Boro nas plantas. Disponível em: http://www.fca.unesp/intranet/helio.php Acessado em 29/11/2009.

INMET. Oitavo Distrito de Meteorologia $\left(8^{\circ}\right.$ DISME). Seção de observação e meteorologia aplicada - SEMA. Porto Alegre, relatório $\mathrm{n}^{\circ}$ 027/2008, protocolado sob $n^{\circ} 4294,24 / 03 / 2008,4$ p.

LA TORRACA, S. M. et al. Recrutamento e exportação de nutrientes por Pinus elliottii var. elliottii em um latossolo vermelho escuro na região de Agudos, SP. IPEF, Piracicaba: n. 27, p. 41-47, 1984.

MARTINEZ, H. E. P. et al. Micronutrientes em Pinus caribea Morelet III. Níveis internos de ferro, manganês e zinco, sob suficiência e sob omissão. Pesquisa Agropecuária Brasileira, Brasília, v. 27, n. 9, p. 1339-1353, set. 1992.

MARENCO, R. A.; LOPES, N. F. Fisiologia vegetal: fotossíntese, respiração, relações hídricas e nutrição mineral. Viçosa: Artmed, 2005. 451 p.

MIYAZAWA, M. et al. Análises Químicas de Tecido Vegetal. In: SILVA, F. C. (Org.). Manual de analises químicas de solos, plantas e fertilizantes. Rio de Janeiro: EMBRAPA SOLOS. 1999. p. 171 $-223$.

MOTTA, F. S. et al. Zoneamento agrícola do Rio Grande do Sul e Santa Catarina: normas agro- climáticas. Pelotas: Ministério da Agricultura, 1971. $80 \mathrm{p}$.

PEREIRA, J. C. et al. Estimativa do conteúdo de nutrientes em um povoamento de Acacia mearnsii De Wild. no Rio Grande do Sul - Brasil. Revista Árvore, Viçosa, v. 24, n. 2, p. 193 - 199, 2000.

SCHUMACHER M. V. et al. Quantificação da biomassa e nutrientes no primeiro desbaste de um povoamento de Pinus taeda L. no estado do Rio Grande do Sul. Relatório de pesquisa. 2007

SCHUMACHER, M. V. et al. Estoque de carbono em florestas de Pinus taeda L. e Acacia mearnsii De Wild. plantadas no estado do Rio Grande do Sul Brasil. As Florestas e o Carbono. Curitiba: UFPR, p. 141-152. 2002.

STAPE, J. L. Production ecology of clonal Eucalyptus plantatins in northeastern Brazil. 2005. 225 f. Tese - Colorado State University, Fort Collins. 2005.

STRECK, E. V. et al. Solos do Rio Grande do Sul. 2. ed. Porto Alegre: Emater-RS, 2008. 107 p.

TEDESCO, M. J. et al. Análise de solo, plantas e outros materiais. Porto Alegre: Departamento de Solos, UFRGS, 1995. 118 p. (Boletim Técnico).

VALERI, S. V. et al. Exportação de biomassa de povoamentos de Pinus taeda L. desbastados em diferentes idades. Floresta, Curitiba, v. 19, n. 1 e 2, p. 23-29, 1989a.

VALERI, S. V. et al. Exportação de nutrientes de povoamentos de Pinus taeda L. desbastados em diferentes idades. Floresta, Curitiba, v. 19, n. 1 e 2, p. 62-68, 1989b.

VAN LEAR, D. H. et al. Biomass and nutrient content of a 41-year-old loblolly pine (Pinus taeda L.) plantation on a site in South Carolina. Forest Science, v. 30, n. 2, p. 395-404, 1984. 